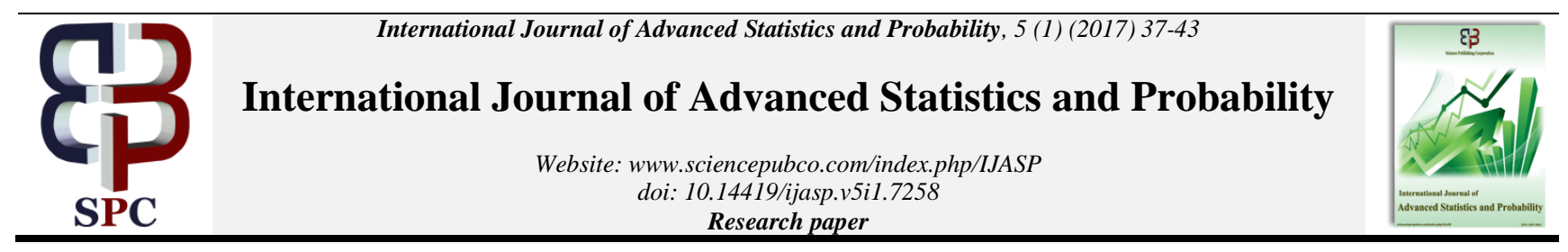

\title{
Analysis of semi-parametric single-index models by using MAVE-method based on some kernel functions
}

\author{
Fayyadh Abdulla Ali ${ }^{1 *}$, Tareq Azeez Salih ${ }^{2}$ \\ ${ }^{1}$ Asst. Prof-Statistic department. ,Administration and Economics college, Wasit university, Iraq \\ ${ }^{2}$ Lecturer-Statistic deptartment. ,Administration and Economics college, Wasit university, Iraq \\ *Corresponding author E-mail:
}

\begin{abstract}
In this paper, we used many forms of kernel functions with minimum average variance estimation (MAVE) method [Xia2002] we called the proposed methods (MAVE-Biweight), (MAVE-Epanechnikov ) and .(MAVE-Gaussian ) for estimation the parameters and the link function of the single - index model (SIM) comparing with other methods of estimation . to evaluate the performing of the various methods s simulation and a real data have been used, conclusions showed that the (MAVE- Gaussian) method in this paper gave better results compared with other methods depending on the mean squared error (MSE) and mean Absolute error (MAE) criterion for comparison.
\end{abstract}

Keywords: Single-Index Model; MAVE Method; Kernel Function; Bandwidth Parameter; Curse of Dimensionality.

\section{Introduction}

SIMs are widely used in sciences of applied quantitative. SIMs looking for a single linear combination of Xs variables that can catch information on a relation between $\mathrm{Y}$ and $\mathrm{X}$, to avoid the curse of dimension specifically the model (SIM) can be written as

$Y=g\left(X^{T} \beta\right)+\epsilon$

$\mathrm{E}\left[\mathrm{Y} \mid \mathrm{X}=\mathrm{x}_{\mathrm{i}}\right]=\mathrm{E}\left[\mathrm{Y} \mid \mathrm{X}^{\mathrm{T}} \beta\right]=\mathrm{P}\left[\mathrm{Y} \mid \mathrm{X}^{\mathrm{T}} \beta\right]=\mathrm{g}\left(\mathrm{X}^{\mathrm{T}} \beta\right)$

Where $E[\in \mid X]=E\left[\epsilon \mid X^{T} \beta\right]=0$ almost surely, $g\left(X^{T} \beta\right)$ is unknown .link function and $\beta$ is vector of parameters such that $\| \beta$ $\|=1$ or $\beta^{\mathrm{T}} \beta=1$, an, and the first component is positive for identification model . Estimation of (SIM) is very important in theory and in practice, In the last years many papers [Tong, $\mathrm{Li}$ and Zhu(2002) ; Yu and .Ruppert (2002) ; Yin and cook ,(2005) ; Delecrax , Hristache and patilea (2006)] have investigated the estimation of the parametric index $\beta$ with focusing on root $-\mathrm{n}$ estimability and efficiency issues. There are three type of methods have been proposed to estimate $\beta$ in the literature ,the first one that include the average derivative estimation method [Hardel and Stoker , (1989)] , the structure adaptive method [Hristach et al, (2002)] and the outer product of gradients (OPG) method [Xia et al ,(2002)], the second type contains methods that estimate $g$ and $\beta$ at same time whereas the third type used regressing $\mathrm{X}$ on $\mathrm{Y}$ instead of regressing Yon $\mathrm{X}$ and were originally suggested dealing with sufficient dimension reduction (SDR) .(['1 ] , [4] , [7] , [16]) . In This paper used the second type from methods it is MAVE method with some kernel functions (Biweight, Epanechnikov and Gaussian) to obtain three methods (MAVE - Biweight, MAVEEpanechnikov and MAVE -Gaussian) for analysis the semiparametric single - index models.
The objective of this paper is identify the best method based on the Two comparison criterion mean squared error (MSE) and mean absolute error (MAE)

The reminder of this paper is arranged as follows. In section 2, a brief review with advantages of semi-parametric single index models and a brief explain for kernel functions with bandwidth parameter. MAVE method reviewed in section 3. A simulation studies are conducted under different setting and the real data and applications of methods are recorded at section 4 and at section 5 the conclusions are summarized.

\section{The semi-parametric single -index model (SSIM)}

Most estimation problems contain both unknown. Finite - dimensional parameter $(\beta)$ and an unknown link function $g\left(X^{\mathrm{T}} \beta\right)$. These kinds of models are. Called "semi-parametric", the linearity assumption $\mathrm{X}^{\mathrm{T}} \beta$ is still valid but no additional assumption is made related to the error term in other a specific link function is not assumed in the model.

In statistic, the technique of regression analysis includes modeling and analysis many variables. it focus on the relationship between a dependent variable and one or more independent variables. More specifically regression analysis applied for estimating the conditional expectation of the dependent variable given the independent variables.

In multiple linear regression models, the conditional mean relationship between the response and each of the predictions is assumed linear. The most flexible models do not make any assumption about the form of the p-variate function. The problem is to fit a $\mathrm{p}$-dimensional surface to the observed data $\{(x \mathrm{iT}, y \mathrm{i}), \mathrm{i}=1,2 \ldots$ $\mathrm{n}\}$. An obvious approach is trying for generalizing the univariate smoothing techniques. There is a sveral problems appear called "curse of dimensionality". A popular way to beat the dimensionality problem is to first project all covariates on to a linear space spanned. by the covariates and then to fit a non-parametric curve. 
To their linear combinations this is lead to the single - index model (SIM):

$y_{\mathrm{i}}=\mathrm{g}\left(x_{\mathrm{i}}^{\mathrm{T}} \beta\right)+\epsilon_{\mathrm{i}}, \mathrm{i}=1,2, \ldots, \mathrm{n}$

Where $\mathrm{g}\left(x_{\mathrm{i}}^{\mathrm{T}} \beta\right)$ is a smooth unknown function, $x_{\mathrm{i}}$ is a $\mathrm{p} \times 1$ vector of covariates, $\beta=\left(\beta_{1}, \beta_{2}, \ldots, \beta_{p}\right)^{\mathrm{T}}$ is a $\mathrm{p} \times 1$ vector of parameters and $\epsilon_{i}$.white noise identical .independent normal distribution with unknown variance $\sigma^{2}$ and mean zero.([6] ,[10],[13],[14]).

\subsection{The advantages of semi-parametric single - index model (SSIM)}

1) The semi-parametric single - index model can avoid the problem of .error distribution misspecification.

2) The (SSIM) is more general than the binary choice model.

3) The response variable (Y) can be discrete or continuous in semi-parametric single - index model.

4) The (SSIM) is an alternative approach .designed to mitigate effects arising from the curse of dimensionality.

5) A single - index model dose not .assume that $\mathrm{g}(x \mathrm{iT} \beta)$ is known. And hence it is more flexible and. less restrictive than parametric models for conditional mean functions, such as linear models and binary probit models, use of a semi-parametric single - index model reduces the risk obtaining misleading results.

6) A single - index model avoids the curse of dimensionality because the index XT $\beta$ aggregates the dimension of X . At the same time $\beta$ can be estimated with same rate of convergence $\mathrm{n}^{-\frac{1}{2}}$ that is achieved in a parametric model.

7) in non- parametric estimation does not .permit extrapolation . it does not provide predictions of $\mathrm{E}[\mathrm{Y} \mid \mathrm{X}=\mathrm{x}]$ at points $\mathrm{X}$ That are not provide in the support of $X$. this is a serious .draw back in policy analysis and forecasting. A semiparametric single -index model by contrast permits extrapolation within limits it yields predictions of $\mathrm{E}[\mathrm{Y} \mid \mathrm{X}=\mathrm{x}]$ at values of $x$ that are not in the support of $\mathrm{X}$ but are in the support of XT $\beta$. ([4],[10],[11],[13])

\subsection{Kernel function selection}

In nonparametric functional estimation , the kernel function analysis with different weight to each data point the weights are depending on the bandwidth and the estimator that are used the function $\mathrm{k}($.$) is generally a symmetric probability density functions$ satisfied the conditions:

1) $\int_{-\infty}^{\infty} \mathrm{k}(\mathrm{u}) \mathrm{du}=1$ is pdf, A kernel function $\mathrm{k}(\mathrm{u}): \mathrm{R} \rightarrow \mathrm{R}$ is any function which satisfies This condition .

2) kernel function is symmetric, $\mathrm{k}(\mathrm{u})=\mathrm{k}(-\mathrm{u})$

3) $\int_{-\infty}^{\infty} \mathrm{uk}(\mathrm{u}) \mathrm{du}=0$

4) $\int_{-\infty}^{\infty} u^{2} k(u) d u=\mu_{2}(k) \neq 0$, The moments of a kernel.

5) $\mathrm{k}(\mathrm{u}) \geq 0$, Anon-negative kernel for all $\mathrm{u}$ when $\mathrm{k}(\mathrm{u})$ is a kernel function, commonly used kernel functions include the Gaussian, the Tricube, Uniform, Biweight, Cosine, Triweight and Epanechnikove kernel function. Gaussian kernel , Biweight kernel and Epanechnikove kernel function are chosen .([2],[3],[11])

Common second-order kernels are listed in the following table.

Table 1: Common Second-Order Kernels

\begin{tabular}{ll}
\hline kernel & Equation \\
\hline Uniform & $\mathrm{K}(\mathrm{u})=\frac{1}{2} 1(|\mathrm{u}| \leq 1)$ \\
Epanechnikov & $\mathrm{K}(\mathrm{u})=\frac{3}{4}\left(1-\mathrm{u}^{2}\right) 1(|\mathrm{u}| \leq 1)$ \\
Biweight & $\mathrm{K}(\mathrm{u})=\frac{15}{16}\left(1-\mathrm{u}^{2}\right)^{2} 1(|\mathrm{u}| \leq 1)$ \\
Triweight & $\mathrm{K}(\mathrm{u})=\frac{35}{32}\left(1-\mathrm{u}^{2}\right)^{3} 1(|\mathrm{u}| \leq 1)$ \\
Gaussian & $\mathrm{K}(\mathrm{u})=\frac{1}{\sqrt{2 \pi}} \exp \left(\frac{-\mathrm{u}^{2}}{2}\right) 1(|\mathrm{u}| \leq \infty)$ \\
Tricube & $\mathrm{K}(\mathrm{u})=\frac{70}{81}\left(1-|\mathrm{u}|^{3}\right)^{3} 1(|\mathrm{u}| \leq 1)$ \\
Cosine & $\mathrm{K}(\mathrm{u})=\frac{\pi}{4} \cos \left(\frac{\pi}{2} \mathrm{u}\right) 1(|\mathrm{u}| \leq 1)$ \\
\hline
\end{tabular}

\subsection{Bandwidth parameter selection}

The bandwidth parameter. also called the smooth parameter (h), controls for the smoothing level of the estimation . (h) Plays very important turn at the performance for kernel estimators. different methods like the cross-validation, penalized functions, , bootstrap , etc.. have been developed to obtain the optimal bandwidths . The cross -validation method in general is preferred due to it is easier in computing and implementation .structure for any regression model the bandwidth value which minimizes the cross-validation $(\mathrm{CV})$ function with a non-negative weight function $\mathrm{w}\left(\mathrm{X}^{\mathrm{T}} \beta\right)$ given as:

$\mathrm{CV}(\mathrm{h})=\mathrm{n}^{-1} \sum_{\mathrm{i}=1}^{\mathrm{n}}\left[\mathrm{Y}_{\mathrm{i}}-\widehat{\mathrm{g}}_{-\mathrm{i}}\left(\mathrm{X}_{\mathrm{i}}^{\mathrm{T}} \widehat{\beta}\right)\right]^{2}$

Is considered the optimal one. The (CV) function contains the leave-one-out kernel defined as follows.

$$
\hat{g}_{-i}\left(X_{i}^{T} \widehat{\beta}\right)=\frac{\sum_{i \neq j}^{n} Y_{i} K_{h}\left(X_{i}^{T} \widehat{\beta}-X_{j}^{T} \widehat{\beta}\right)}{\sum_{i \neq j}^{n} K_{h}\left(X_{i}^{T} \widehat{\beta}-X_{j}^{T} \widehat{\beta}\right)}
$$

It used to estimate our mean function, a Nadaraya - watson kernel smoothing.

The leave-one-out estimator is obtained. by leaving out the observations $\mathfrak{i}$

(The concerned observations $x_{\mathrm{i}}$ and $y_{\mathrm{i}}$ ) from. The data each time for satisfying. The unbiased estimate of the bandwidth parameter (h). The procedure is replicated (n) times. (For all observations). The final optimal bandwidth value. Required for the kernel estimation .is the mean of all these values computed. The bandwidth that minimizes the cross-validation function also minimizes the MSE is a performance criterion of an estimator. ([2], [9], [12]). 3.

\section{Minimum average variance estimation (MAVE) method}

For estimation of single - index model $E[Y \mid X=x]=g\left(X^{T} \beta\right)$ proceed in two step:

First, the coefficient vector $\beta$ has to estimation methods to calculate the coefficients for discrete and continuous variables will be covered depthly later.

Secondly, for estimation the unknown link function $g\left(X^{\mathrm{T}} \beta\right)$ by non-parametrically regression the dependent variable $\mathrm{Y}$ on the fitting index $X^{\mathrm{T}} \widehat{\beta}$ where $\widehat{\beta}$ is the coefficient vector. we estimated the kernel estimator. [11]

In (2002) Xia, Tong, $\mathrm{Li}$ and $\mathrm{Zhu}$ proposed a general estimation method termed minimum average variance estimation (MAVE) for semi-parametric models.

Let $\left\{\mathrm{x}_{\mathrm{i}}, \mathrm{y}_{\mathrm{i}} ; \mathrm{i}=1,2, \ldots, \mathrm{n}\right\}$ be a random samples from model (1) the basic concept of our estimation method is to linearly approximation the smooth link function $\mathrm{g}\left(\mathrm{X}^{\mathrm{T}} \beta\right)$ and estimation $\beta$ by minimizing the overall approximation errors. The single - index model (1) is a special case of what they considered and we can estimate it as follows. Assuming function $g\left(X^{\mathrm{T}} \beta\right)$ and parameter $\beta$. Then the Taylor. expansion of $\mathrm{g}\left(x_{\mathrm{i}}^{\mathrm{T}} \beta\right)$ at $\mathrm{g}\left(\mathrm{x}^{\mathrm{T}} \beta\right)$ is:

$g\left(x_{i}^{T} \beta\right) \approx a+b\left(x_{i}-x\right)^{T} \beta$

Where $a=g(x \mathrm{~T} \beta), b=g /(x \mathrm{~T} \beta)$ with fixed $\beta$ the local estimator of conditional variance is:

$\sigma_{\mathrm{n}}^{2}(\mathrm{x} \mid \beta)=\min \left\{\mathrm{nf}_{\beta}(\mathrm{x})\right\}^{-1} \sum_{\mathrm{i}=1}^{\mathrm{n}}\left\{\mathrm{Y}_{\mathrm{i}}-\left\{\mathrm{a}+\mathrm{b}(\mathrm{Xi}-\mathrm{x})^{\mathrm{T}} \beta\right\}^{2} \cdot \mathrm{kh}_{\mathrm{h}}\right.$

$\left\{\left(X_{i}-x\right)^{\mathrm{T}} \beta\right\}$

Where $\hat{\mathrm{f}}_{\beta}(\mathrm{x})=\mathrm{n}^{-1} \sum_{\mathrm{i}=1}^{\mathrm{n}} \operatorname{kh}\left\{(\mathrm{xi}-\mathrm{x})^{\mathrm{T}} \beta\right\}$ where $\mathrm{k}$ is density function, $h$ is the bandwidth and $k_{h}(u)=k_{h}(u / h) / h$. 
$\sigma_{\mathrm{n}}^{2}(\mathrm{x} \mid \beta)$ can also be understood, the best approximation minimize the overall departure at all $\mathrm{x}=x_{j}, j=1,2, \ldots, \mathrm{n}$, Thus , $\beta$ estimator is to minimize:

$$
Q_{n}(\beta)=\sum_{i=1}^{n} \sigma_{n}^{2}\left(x_{j} \mid \beta\right)
$$

The basic algorithm. for estimation $\mathrm{g}($.) in the single - index model is based on observing that

$$
\beta=\operatorname{argmin} E\left[Y-g\left(X^{T} \beta\right)\right]^{2}
$$

Subject to $\beta^{\mathrm{T}} \beta=1$, by condition. on $\mathrm{X}^{\mathrm{T}} \beta$ we see that (5) equals

$\mathrm{E}_{\mathrm{u}} \sigma_{\beta}^{2}\left(\mathrm{x}^{\mathrm{T}} \beta\right)$ where $\sigma_{\beta}^{2}\left(\mathrm{x}^{\mathrm{T}} \beta\right)=\mathrm{E}\left[\left(\mathrm{Y}-\mathrm{g}\left(\mathrm{X}^{\mathrm{T}} \beta\right)\right)^{2} \mid \mathrm{X}^{\mathrm{T}} \beta=\mathrm{u}\right]$

$\mathrm{E}_{\mathrm{u}} \sigma_{\beta}^{2}\left(\mathrm{x}^{\mathrm{T}} \beta\right)$ where $\sigma_{\beta}^{2}\left(\mathrm{x}^{\mathrm{T}} \beta\right)=\mathrm{E}\left[\left(\mathrm{Y}-\mathrm{g}\left(\mathrm{X}^{\mathrm{T}} \beta\right)\right)^{2} \mid \mathrm{X}^{\mathrm{T}} \beta=\mathrm{u}\right]$

If follows that:

$$
\mathrm{E}\left[\mathrm{Y}-\mathrm{g}\left(\mathrm{X}^{\mathrm{T}} \beta\right)\right]^{2}=\mathrm{E}_{\mathrm{u}} \sigma_{\beta}^{2}\left(\mathrm{x}^{\mathrm{T}} \beta\right)
$$

Therefore, minimizing (5) is equivalent to:

$$
\beta=\operatorname{argmin} \mathrm{E}_{\mathrm{u}} \sigma_{\beta}^{2}\left(\mathrm{x}^{\mathrm{T}} \beta\right)
$$

The conditional expectation in (6) is now approximation by the sample analogue for $\mathrm{x}_{\mathrm{i}}$, we have the following local linear approximation.

$y_{i}-g\left(x_{i}^{T} \beta\right) \approx y_{i}-g\left(x^{T} \beta\right)-g\left(x^{T} \beta\right) \cdot x_{i j}^{T} \beta$

Where $x_{i j}=x_{i}-x_{j}$.

Following. The idea of local linear smoothing, we may estimate $\sigma_{\beta}^{2}\left(\mathrm{x}^{\mathrm{T}} \beta\right)$ by:

$$
\sigma_{\beta}^{2}\left(x^{T} \beta\right)=\min _{a, b} \sum_{i=1}^{n}\left\{Y_{i}-a-b X_{i j}^{T} \beta\right\}^{2} \cdot w_{i} 0
$$

Here, $w_{i 0} \geq 0, i=1,2, \ldots, n$, are some weights. With $\sum_{i=1}^{n} w_{i 0}=1$ ,typically .centering at $\mathrm{x}$. By (6) and (7) our estimation procedure is to minimize.

$$
\frac{1}{n} \sum_{j=1}^{n} \sum_{i=1}^{n}\left\{Y_{i}-a_{j}-b_{j} X_{i j}^{T} \beta\right\}^{2} \cdot w_{i j} n
$$

With .respect to $\left(a_{j}, b_{j}\right)$ and $\beta$. if the kernel smoothing is used with kernel function $\mathrm{k}(\mathrm{u})$ and bandwidth $(\mathrm{h})$, Then the weight function $w_{i j}=k_{h}\left(X_{i j}^{T}\right)$, where $w_{i j}=\frac{k_{h}\left(X_{i j}^{T} \beta\right)}{\sum_{i=1}^{n} k_{h}\left(X_{i j}^{T} \beta\right)}$

We call the estimation procedure. The Minimum Average Variance Estimation (MAVE) method. ([5], [8], [15], [16]).

Our estimation procedure for $\beta$ and $g\left(x^{\mathrm{T}} \beta\right)$ is described. in details as follows:

Step (0): initialization step obtain an initial estimate of $\beta$, let $\widehat{\beta}^{(0)}$ be an estimator of the OLS method or arbitrary.
Step (1): put $\widehat{\beta}^{(0)}=\beta$ and calculate the solution of $\left(a_{j}, b_{j}\right)$.

$$
\left(\begin{array}{l}
\hat{a}_{j} \\
\hat{b}_{j}
\end{array}\right)=\left[\sum_{i=1}^{n} k_{h}\left(X_{i j}^{T} \beta\right)\left(\begin{array}{c}
1 \\
\frac{x_{i j}^{T} \beta}{h}
\end{array}\right)\left(\begin{array}{c}
1 \\
\frac{x_{i j}^{T} \beta}{h}
\end{array}\right)^{T}\right]^{-1} \sum_{i=1}^{n} k_{h}\left(X_{i j}^{T} \beta\right)\left(\begin{array}{c}
1 \\
\frac{x_{i j}^{T} \beta}{h}
\end{array}\right) Y_{i}
$$

Step (2): calculate

$\left.\widehat{\beta}=\left\{\sum_{i, j}^{n} k_{h}\left(X_{i j}^{T} \beta\right)\left(\hat{b}_{j}\right)^{2} \cdot X_{i j} X_{i j}^{T}\right\}^{-1} \cdot \sum_{i, j}^{n} k_{h}\left(X_{i j}^{T} \beta\right) \hat{b}_{j} X_{i j}\left(Y_{i}-\hat{a}_{j}\right)\right\}$

And

$\widehat{\beta}^{\mathrm{MAVE}}=\operatorname{sign}\left(\widehat{\beta}_{1}\right) \frac{\widehat{\beta}}{\|\widehat{\beta}\|}$

Step (3): Repeat step (1) and step (2) until the iteration process converges, the final vector $\beta$ is The MAVE estimator for $\hat{\beta}^{(0)}$.

$\mathrm{Xia}$ and Tong (2006) proved that the. estimator produced by the algorithm can a chive root $-\mathrm{n}$ consistency and has the same. asymptotic distribution as the estimator of Hardle et al (1993)

\section{Numerical studies}

In This section we illustrate the performance of methods (MAVEBiweight, MAVE -Epanechnikov and MAVE-Gaussian ) by Three simulation studies and real data for Iraq stock exchange analysis based on (R-Package) .

\subsection{Simulation}

Experiment1. In this experiment we simulate 200 datasets consisting of sample size $n=25,100$ observation from the following model.

$\mathrm{Y}=\sin \left(\mathrm{X}^{\mathrm{T}} \beta\right)+\epsilon$

Where $X=\left(x_{1}, x_{2}, x_{3}, x_{4}, x_{5}\right)^{T}, \beta=(1,1,1,1,1)^{T} / \sqrt{5}, x_{i}$ are iid $\sim \mathrm{N}\left(0, \sigma^{2}\right), \epsilon \sim \mathrm{N}(0,1)$ and correlation between $\mathrm{x}_{\mathrm{i}}$ and $\mathrm{x}_{\mathrm{j}}$, is $\rho^{|\mathrm{i}-\mathrm{j}|}$ for $\mathrm{i}, \mathrm{j}=1,2,3,4,5$. With two values of $\rho$ were explored 0.2 and 0.9 with $\epsilon$ and $X$ are independent.

Experiment 2 in this experiment we simulate 200 datasets consisting of sample size $n=25,100$ observation from the following model with homoscedastic errors.

$Y=2 \sin \left(X^{T} \beta\right)+\exp \left(2 X^{T} \beta\right)+0.5 \epsilon$

Where $X=\left(x_{1}, x_{2}, x_{3}, x_{4}, x_{5}, x_{6}\right)^{T}, \beta=(1,1,1,1,1,0)^{T} / \sqrt{5}, x_{i}$ are iid $\sim \mathrm{N}\left(0, \sigma^{2}\right), \epsilon \sim \mathrm{N}(0,1)$ and correlation between $\mathrm{xi}$ and $\mathrm{xj}$, is $\rho^{|\mathrm{i}-\mathrm{j}|}$ for $\mathrm{i}, \mathrm{j}=1,2,3,4,5,6$. With Two values of $\rho$ were explored 0.2 and 0.9 with $\epsilon$ and $X$ are independent.

Experiment 3. In this experiment we simulate 200 datasets consisting of sample size

$\mathrm{n}=25,100$ observation from the following model.

$\mathrm{Y}=1+2\left(\mathrm{X}^{\mathrm{T}} \beta+3\right) \log \left(3\left|\mathrm{X}^{\mathrm{T}} \beta\right|+1\right)+\epsilon$

WhereX $=\left(\mathrm{x}_{1}, \mathrm{x}_{2}, \mathrm{x}_{3}, \mathrm{x}_{4}, \mathrm{x}_{5}, \mathrm{x}_{6}, \mathrm{x}_{7}\right)^{\mathrm{T}}, \beta=(0.4,-0.4,0.8,-0.2,0,0,0)^{\mathrm{T}}$ , $x_{i}$ are iid $\sim \mathrm{N}\left(0, \sigma^{2}\right), \epsilon \sim \mathrm{N}(0,1)$ and correlation between $x i$ and $x j$ , is $\rho^{|\mathrm{i}-\mathrm{j}|}$ for $\mathrm{i}, \mathrm{j}=1,2,3,4,5,6,7$. With two values of $\rho$ were explored 0.2 and 0.9 with $\epsilon$ and $X$ are independent. 
Table 2: Amse For Coefficients B Estimated by Methods (Mave-Biwieght, Mave-Epanechnikov and Mave - Gaussian) Based on the Model in Experiment 2 for Sample Size, $N=25,100$ with $P=0.2,0.9$ and $P=5$.

\begin{tabular}{|c|c|c|c|c|c|c|c|c|}
\hline$\rho$ & sample size & methods & & $\widehat{\beta}_{1}$ & $\widehat{\beta}_{2}$ & $\widehat{\beta}_{3}$ & $\widehat{\beta}_{4}$ & $\widehat{\beta}_{5}$ \\
\hline \multirow{6}{*}{0.2} & \multirow{6}{*}{25} & \multirow{3}{*}{ MAVE-B } & (Average) $\widehat{\beta}$ & 0.43730 & 0.51004 & 0.42394 & 0.45107 & 0.40672 \\
\hline & & & AMSE & 0.13259 & 0.10707 & 0.12220 & 0.11685 & 0.11209 \\
\hline & & & (Average) $\widehat{\beta}$ & 0.44845 & 0.44731 & 0.45129 & 0.46509 & 0.42287 \\
\hline & & MAVE-E & (Average) $\widehat{\beta}$ & 0.45179 & 0.44625 & 0.45506 & 0.46736 & 0.41379 \\
\hline & & \multirow[t]{2}{*}{ MAVE-G } & AMSE & 0.04670 & 0.04592 & 0.04180 & 0.05297 & 0.04708 \\
\hline & & & (Average) $\widehat{\beta}$ & 0.54414 & 0.38704 & 0.32959 & 0.47440 & 0.46947 \\
\hline \multirow{5}{*}{0.9} & \multirow{5}{*}{25} & \multirow[t]{2}{*}{ MAVE-B } & AMSE & 0.15749 & 0.21327 & 0.24989 & 0.23489 & 0.14976 \\
\hline & & & (Average) $\widehat{\beta}$ & 0.45664 & 0.44839 & 0.42537 & 0.44849 & 0.45642 \\
\hline & & \multirow[t]{2}{*}{ MAVE-E } & AMSE & 0.09681 & 0.16980 & 0.17228 & 0.16512 & 0.10290 \\
\hline & & & (Average) $\widehat{\beta}$ & 0.47592 & 0.42583 & 0.43109 & 0.44991 & 0.45155 \\
\hline & & \multirow[t]{2}{*}{ MAVE-G } & AMSE & 0.09500 & 0.18556 & 0.16934 & 0.17596 & 0.09634 \\
\hline \multirow{6}{*}{0.2} & \multirow{6}{*}{100} & & (Average) $\widehat{\beta}$ & 0.41724 & 0.44376 & 0.46495 & 0.51013 & 0.39058 \\
\hline & & \multirow[t]{2}{*}{ MAVE-B } & AMSE & 0.14655 & 0.14493 & 0.11837 & 0.13704 & 0.12885 \\
\hline & & & (Average) $\widehat{\beta}$ & 0.44295 & 0.44754 & 0.44658 & 0.46118 & 0.43745 \\
\hline & & MAVE-E & AMSE & 0.01467 & 0.01120 & 0.01331 & 0.01666 & 0.01176 \\
\hline & & \multirow[t]{2}{*}{ MAVE-G } & AMSE & 0.01440 & 0.01215 & 0.01341 & 0.01706 & 0.01175 \\
\hline & & & (Average) $\hat{\beta}$ & 0.42333 & 0.32393 & 0.58982 & 0.52445 & 0.30482 \\
\hline \multirow{5}{*}{0.9} & & \multirow[t]{2}{*}{ MAVE-B } & AMSE & 0.14855 & 0.21529 & 0.24252 & 0.24470 & 0.15464 \\
\hline & & & (Average) $\hat{\beta}$ & 0.44599 & 0.44144 & 0.46386 & 0.45112 & 0.43303 \\
\hline & 100 & MAVE-E & AMSE & 0.04707 & 0.08485 & 0.07157 & 0.07803 & 0.04301 \\
\hline & & & (Average) $\hat{\beta}$ & 0.44425 & 0.44694 & 0.45591 & 0.45031 & 0.43844 \\
\hline & & MAVE-G & AMSE & 0.05032 & 0.08705 & 0.07595 & 0.08052 & 0.04305 \\
\hline
\end{tabular}

Table 3: AMSE for Coefficients B Estimated by Methods (MAVE-Biwieght, MAVE-Epanechnikov and MAVE - Gaussian) Based on the Model in Experiment 1 for Sample Size $N=25,100$ with $\mathrm{P}=0.2,0.9$ and $\mathrm{P}=6$

\begin{tabular}{|c|c|c|c|c|c|c|c|c|c|}
\hline$\rho$ & sample size & methods & & $\widehat{\beta}_{1}$ & $\widehat{\beta}_{2}$ & $\widehat{\beta}_{3}$ & $\widehat{\beta}_{4}$ & $\widehat{\beta}_{5}$ & $\widehat{\beta}_{6}$ \\
\hline \multirow{7}{*}{0.2} & \multirow{7}{*}{25} & \multirow{3}{*}{ MAVE-B } & (Average) $\hat{\beta}$ & 0.43530 & 0.45099 & 0.42675 & 0.45290 & 0.44194 & 0.15668 \\
\hline & & & AMSE & 0.01817 & 0.01774 & 0.01851 & 0.01959 & 0.01926 & 0.04949 \\
\hline & & & (Average) $\hat{\beta}$ & 0.45484 & 0.44343 & 0.44485 & 0.44736 & 0.44087 & 0.06392 \\
\hline & & \multirow[t]{2}{*}{ MAVE-E } & AMSE & 0.00563 & 0.00781 & 0.00666 & 0.00645 & 0.00733 & 0.00947 \\
\hline & & & (Average) $\hat{\beta}$ & 0.45372 & 0.44389 & 0.44551 & 0.44700 & 0.44082 & 0.06685 \\
\hline & & \multirow[t]{2}{*}{ MAVE-G } & AMSE & 0.00546 & 0.00728 & 0.00647 & 0.00636 & 0.00726 & 0.00964 \\
\hline & & & (Average) $\hat{\beta}$ & 0.38431 & 0.52544 & 0.39215 & 0.47639 & 0.41046 & 0.16429 \\
\hline \multirow{4}{*}{0.9} & \multirow{4}{*}{25} & MAVE-B & (Average) $\hat{\beta}$ & 0.45352 & 0.41453 & 0.48001 & 0.41590 & 0.46334 & 0.06624 \\
\hline & & \multirow{2}{*}{ MAVE-E } & AMSE & 0.03831 & 0.08195 & 0.06311 & 0.07267 & 0.04842 & 0.04065 \\
\hline & & & (Average) $\hat{\beta}$ & 0.45494 & 0.41421 & 0.47978 & 0.41314 & 0.46459 & 0.06869 \\
\hline & & \multirow[t]{2}{*}{ MAVE-G } & AMSE & 0.03676 & 0.08167 & 0.06137 & 0.07093 & 0.04758 & 0.03999 \\
\hline \multirow{6}{*}{0.2} & \multirow{6}{*}{100} & & (Average) $\hat{\beta}$ & 0.42820 & 0.45863 & 0.43980 & 0.44838 & 0.45589 & 0.06310 \\
\hline & & \multirow[t]{2}{*}{ MAVE-B } & AMSE & 0.02092 & 0.01977 & 0.02085 & 0.01914 & 0.02005 & 0.02731 \\
\hline & & & (Average) $\hat{\beta}$ & 0.44480 & 0.45143 & 0.44421 & 0.44791 & 0.44469 & 0.05145 \\
\hline & & MAVE-E & AMSE & 0.00126 & 0.00107 & 0.00108 & 0.00113 & 0.00129 & 0.00331 \\
\hline & & \multirow[t]{2}{*}{ MAVE-G } & AMSE & 0.00122 & 0.00104 & 0.00105 & 0.00111 & 0.00125 & 0.00387 \\
\hline & & & (Average) $\hat{\beta}$ & 0.41465 & 0.54081 & 0.36691 & 0.32506 & 0.54070 & 0.05410 \\
\hline \multirow{5}{*}{0.9} & \multirow{5}{*}{100} & \multirow[t]{2}{*}{ MAVE-B } & AMSE & 0.10381 & 0.16461 & 0.16566 & 0.16215 & 0.17670 & 0.09891 \\
\hline & & & (Average) $\hat{\beta}$ & 0.44701 & 0.45064 & 0.43805 & 0.43993 & 0.45746 & 0.04886 \\
\hline & & \multirow[t]{2}{*}{ MAVE-E } & AMSE & 0.00705 & 0.01266 & 0.01427 & 0.01577 & 0.01021 & 0.00814 \\
\hline & & & (Average) $\hat{\beta}$ & 0.44709 & 0.45005 & 0.43821 & 0.44084 & 0.45671 & 0.05082 \\
\hline & & MAVE-G & AMSE & 0.00694 & 0.01240 & 0.01398 & 0.01542 & 0.00993 & 0.00822 \\
\hline
\end{tabular}


Table 4: The Average and Average Mean Squared Error (AMSE) for Coefficients B which are Estimated by Methods (MAVE-Biwieght, MAVEEpanechnikov and MAVE - Gaussian) Based on the Model in Experiment 3 for Sample Size, N=25,100 with P=0.2, 0.9 and $\mathrm{P}=7$.

\begin{tabular}{|c|c|c|c|c|c|c|c|c|c|c|}
\hline$\rho$ & $\begin{array}{l}\text { sample } \\
\text { size }\end{array}$ & methods & & $\widehat{\beta}_{1}$ & $\widehat{\beta}_{2}$ & $\widehat{\beta}_{3}$ & $\widehat{\beta}_{4}$ & $\widehat{\beta}_{5}$ & $\widehat{\beta}_{6}$ & $\hat{\beta}_{7}$ \\
\hline \multirow{7}{*}{0.2} & \multirow{7}{*}{25} & \multirow{3}{*}{ MAVE-B } & (Average) $\hat{\beta}$ & 0.50403 & 0.06336 & .0 .69527 & 0.15367 & 0.29558 & 0.27241 & 0.27079 \\
\hline & & & AMSE & 0.11876 & 0.34889 & 0.27521 & 0.20426 & 0.15983 & 0.13213 & 0.13893 \\
\hline & & & (Average) $\hat{\beta}$ & 0.47524 & 0.12630 & 0.67674 & 0.21132 & 0.28459 & 0.32911 & 0.25736 \\
\hline & & \multirow[t]{2}{*}{ MAVE-E } & AMSE & 0.06281 & 0.39983 & 0.15202 & 0.25486 & 0.13386 & 0.16192 & 0.12006 \\
\hline & & & (Average $) \hat{\beta}$ & 0.47489 & 0.11662 & 0.67567 & 0.20894 & 0.28846 & 0.33253 & 0.25865 \\
\hline & & \multirow[t]{2}{*}{ MAVE-G } & AMSE & 0.06482 & 0.39051 & 0.15760 & 0.25638 & 0.13948 & 0.16481 & 0.12028 \\
\hline & & & (Average) $\hat{\beta}$ & 0.50862 & -0.24808 & 0.80748 & -0.10937 & 0.05614 & 0.10344 & 0.04374 \\
\hline \multirow{5}{*}{0.9} & \multirow{5}{*}{25} & \multirow[t]{2}{*}{ MAVE-B } & AMSE & 0.08078 & 0.13219 & 0.10663 & 0.12197 & 0.09106 & 0.12756 & 0.07520 \\
\hline & & & (Average) $\hat{\beta}$ & 0.42045 & -0.31698 & 0.83119 & -0.16678 & 0.04639 & 0.03083 & 0.03071 \\
\hline & & \multirow[t]{2}{*}{ MAVE-E } & AMSE & 0.03681 & 0.07596 & 0.07010 & 0.05790 & 0.05514 & 0.06106 & 0.04176 \\
\hline & & & (Average) $\hat{\beta}$ & 0.41418 & -0.31556 & 0.83295 & -0.17548 & 0.04734 & 0.03476 & 0.02835 \\
\hline & & \multirow[t]{2}{*}{ MAVE-G } & AMSE & 0.03608 & 0.07927 & 0.05909 & 0.05150 & 0.05237 & 0.05539 & 0.03959 \\
\hline \multirow{7}{*}{0.2} & \multirow{7}{*}{100} & & (Average) $\hat{\beta}$ & 0.45937 & -0.28840 & 0.82163 & -0.12303 & 0.06299 & 0.07807 & 0.07423 \\
\hline & & \multirow[t]{2}{*}{ MAVE-B } & AMSE & 0.08680 & 0.11735 & 0.30022 & 0.04941 & 0.02349 & 0.02198 & 0.02349 \\
\hline & & & (Average) $\hat{\beta}$ & 0.46511 & -0.21177 & 0.82594 & -0.04414 & 0.13369 & 0.14034 & 0.13080 \\
\hline & & \multirow[t]{2}{*}{ MAVE-E } & AMSE & 0.02069 & 0.12686 & 0.06916 & 0.07343 & 0.04367 & 0.04909 & 0.04142 \\
\hline & & & (Average) $\hat{\beta}$ & 0.46472 & -0.21250 & 0.82637 & -0.04733 & 0.13311 & 0.13872 & 0.12955 \\
\hline & & \multirow[t]{2}{*}{ MAVE-G } & AMSE & 0.02112 & 0.12354 & 0.07022 & 0.06934 & 0.04220 & 0.04775 & 0.04053 \\
\hline & & & (Average) $\hat{\beta}$ & 0.42525 & -0.37670 & 0.80106 & -0.18429 & 0.03120 & 0.02013 & 0.01374 \\
\hline \multirow{5}{*}{0.9} & \multirow{5}{*}{100} & \multirow[t]{2}{*}{ MAVE-B } & AMSE & 0.02259 & 0.02473 & 0.01581 & 0.03841 & 0.03878 & 0.04224 & 0.02250 \\
\hline & & & (Average) $\hat{\beta}$ & 0.41291 & -0.38675 & 0.80296 & -0.18630 & 0.01184 & 0.01157 & 0.01364 \\
\hline & & MAVE-E & AMSE & 0.00193 & 0.00490 & 0.00195 & 0.00378 & 0.00296 & 0.00567 & 0.00249 \\
\hline & & & (Average) $\hat{\beta}$ & 0.41506 & -0.38257 & 0.80467 & -0.18198 & 0.01506 & 0.01419 & 0.01754 \\
\hline & & MAVE-G & AMSE & 0.00203 & 0.00538 & 0.00174 & 0.00448 & 0.00343 & 0.00552 & 0.00328 \\
\hline
\end{tabular}

Table 5: AMSE for $\widehat{\mathrm{G}}\left(\mathrm{X}_{\mathrm{i}}^{\mathrm{t}} \widehat{\mathrm{B}}\right)$ Estimated by Methods (MAVE-Biwieght, MAVE-Epanechnikov and MAVE - Gaussian) Sample Size N $=25,100$ with $\rho=$ $0.2,0.9$

\begin{tabular}{|c|c|c|c|c|c|c|c|c|c|c|}
\hline \multirow{2}{*}{ Model } & \multirow{2}{*}{$\mathrm{p}$} & \multirow[b]{2}{*}{ methods } & \multicolumn{2}{|c|}{$\rho=0.2, n=25$} & \multicolumn{2}{|c|}{$\rho=0.2, n=100$} & \multicolumn{2}{|c|}{$\rho=0.9, n=25$} & \multicolumn{2}{|c|}{$\rho=0.9, n=100$} \\
\hline & & & AMSE & AMAE & AMSE & AMAE & AMSE & AMAE & AMSE & AMAE \\
\hline \multirow{3}{*}{1} & \multirow{3}{*}{5} & MAVE-E & 0.009851 & 0.005651 & 0.009699 & 0.0055977 & 0.00972 & 0.00559 & 0.00220 & 0.00534 \\
\hline & & MAVE-G & 0.009919 & 0.005658 & 0.00952 & 0.00555 & 0.00869 & 0.00530 & 0.00861 & 0.00571 \\
\hline & & MAVE-B & 0.42850 & 0.02478 & 0.45480 & 0.02682 & 825.082 & 0.12472 & 121.548 & 0.13056 \\
\hline 2 & 6 & MAVE-E & 0.42837 & 0.02478 & 0.41450 & 0.02383 & 825.076 & 0.12478 & 121.465 & 0.11303 \\
\hline \multirow{3}{*}{3} & \multirow{3}{*}{7} & MAVE-B & 0.27856 & 0.02699 & 0.20045 & 0.02272 & 0.17318 & 0.02077 & 0.13185 & 0.01840 \\
\hline & & MAVE-E & 0.27955 & 0.02706 & 0.20086 & 0.02272 & 0.17067 & 0.02065 & 0.12640 & 0.01815 \\
\hline & & MAVE-G & 0.25173 & 0.02570 & 0.18155 & 0.02186 & 0.19794 & 0.02570 & 0.13030 & 0.01844 \\
\hline
\end{tabular}

According to the AMSE for the coefficients $\hat{\beta}$, from Table 2,3, and 4 .In general, and for different experiments we observed that in the majority of the estimated coefficients, and According to the AMSE and AMAE for the link function $\hat{g}\left(\mathrm{X}_{\mathrm{i}}^{\mathrm{T}} \widehat{\beta}\right)$ from table 5. The MAVE-G method have a lower AMSE and a lower AMAE than the MAVE-E and MAVE-B method.

\subsection{Real data}

To illustrate the performance of our methods from through analysis of the Iraq stock exchange data, the data set in our consist of $\mathrm{n}=27$ companies, 21 banks sector, 4 Insurance sector and 2 investment sector. Through of average Interval (2008-2011) based on companies guide and it is a variable in the R-Package.

The response variable $\left(\mathrm{y}_{\mathrm{i}}\right)$ is Earning per share (Iraqi Dinars (ID)) and The selected There are seven explanatory variables.

$x_{1}$ - Share Turnover Ratio (\%)

$x_{2}$ - Owner ship Rate (\%) $x_{3}$ - Interest Repetition (time)

$x_{4}$ - Trading Rate $(\%)$

$x_{5}$ - Book Value (ID)

$x_{6}$ - Annual closing price (ID)

$x_{7}$ - Annual Average price

We estimate vector of parameters $(\beta)$ and link function $g\left(X^{\mathrm{T}} \beta\right)$ for semi-parametric singl - index model between The Earning per share $\left(\mathrm{yi}_{\mathrm{i}}\right)$ and seven independent variables for predictor to earning per share in Iraq stock exchange from The following semiparametric single - index model :

$y_{i}=g\left(x_{i 1} \beta_{1}+x_{i 2} \beta_{2}+x_{i 3} \beta_{3}+x_{i 4} \beta_{4}+x_{i 5} \beta_{5}+x_{i 6} \beta_{6}+\right.$ $\left.\mathrm{x}_{\mathrm{i} 7} \beta_{7}\right)+\epsilon_{\mathrm{i}}$

According to Real data listed in the table below. Based on companies guide of Iraq stock exchange. 
Table 6: A Real Data for Iraq Stock Exchange of Average Interval (2008-2011)

\begin{tabular}{|c|c|c|c|c|c|c|c|c|}
\hline $\mathrm{n}$ & $\mathrm{Yi}$ & $\mathrm{X} 1$ & $\mathrm{X} 2$ & $\mathrm{X} 3$ & $\mathrm{X} 4$ & $\mathrm{X} 5$ & X6 & $\mathrm{X7}$ \\
\hline 1 & 0.095 & 12.028 & 43.525 & 21.6475 & 1.785 & 1.36275 & 1.315 & 1.41 \\
\hline 2 & 0.20475 & 17.7275 & 14.7775 & 12.9375 & 1.1425 & 1.26125 & 2.48 & 2.46 \\
\hline 3 & 0.0055 & 41.3725 & 52.24 & 156.3275 & 2.0125 & 1.005 & 0.94 & 0.96 \\
\hline 5 & 0.1315 & 19.4175 & 33.5125 & 10.585 & 1.4475 & 1.2115 & 1.21 & 1.205 \\
\hline 6 & 0.045 & 6.395 & 51.3475 & 64.7852 & 2.015 & 1.0975 & 0.8575 & 0.96 \\
\hline 7 & 0.145 & 2.365 & 28.805 & 25.51 & 1.405 & 1.38 & 3.075 & 3.11375 \\
\hline 8 & 0.15175 & 0.775 & 14.3 & 120.24 & 1.155 & 1.035 & 5.625 & 5.9625 \\
\hline 9 & 0.02925 & 76.325 & 69.1925 & 111.5175 & 2.5275 & 1.1075 & 0.8575 & 0.9175 \\
\hline 10 & 0.09925 & 11.16 & 33.59 & 14.0325 & 1.38 & 1.17375 & 1.145 & 1.1 \\
\hline 11 & 0.22625 & 10.255 & 32.66 & 17.8325 & 1.3675 & 1.3605 & 2.1975 & 1.8225 \\
\hline 12 & 0.2075 & 16.555 & 24.185 & 8 & 1.2525 & 1.275025 & 1.29 & 1.2125 \\
\hline 13 & 0.185 & 14.705 & 29.0525 & 7.3475 & 1.3775 & 1.28975 & 1.215 & 1.2075 \\
\hline 14 & 0.08075 & 3.7625 & 53.485 & 13.1875 & 2.03 & 1.12 & 0.9125 & 0.915 \\
\hline 15 & 0.1925 & 24.82 & 24.6475 & 12.19 & 1.2575 & 1.3025 & 2.2025 & 2.48875 \\
\hline 16 & 0.1855 & 2.345 & 26.365 & 11.3225 & 1.3175 & 1.39525 & 1.8425 & 1.8025 \\
\hline 17 & 0.14725 & 9.125 & 46.27 & 7.07 & 1.7925 & 1.2035 & 1.005 & 1.06375 \\
\hline 19 & 0.145 & 25.39 & 34.1825 & 8.3375 & 1.49 & 1.375 & 1.575 & 1.465 \\
\hline 20 & 0.08175 & 1.2875 & 32.045 & 4.2425 & 1.365 & 1.09925 & 0.39 & 0.43 \\
\hline 21 & 0.26175 & 0.095 & 33.02 & 1.155 & 1.39 & 1.1075 & 0.2425 & 0.245 \\
\hline 22 & 0.13725 & 24.675 & 80.5375 & 16.3325 & 5.6 & 1.485 & 1.255 & 1.27 \\
\hline 23 & 0.08025 & 16.885 & 94.4175 & 103.56 & 19.1125 & 1.3765 & 2.4875 & 2.7625 \\
\hline 24 & 0.10975 & 8.605 & 89.145 & 14.2925 & 8.4925 & 1.3625 & 1.055 & 1.125 \\
\hline 25 & 0.001925 & 51.2875 & 91.7925 & 45.2225 & 14.97 & 1.053 & 1.21 & 1.275 \\
\hline 26 & 0.09425 & 11.335 & 93.41 & 67.495 & 23.51 & 11.075 & 1.6625 & 1.6735 \\
\hline 27 & 0.05 & 13.0178 & 91.6375 & 27.9075 & 11.9425 & 1.16925 & 1.0875 & 1.0225 \\
\hline
\end{tabular}

Table 7: The Estimated Coefficients $\widehat{B}$ for Single Index Model which are Estimated by Methods (MAVE-Biwieght, MAVE- Epanechnikov and MAVEGaussian) Based on Real Data

\begin{tabular}{lcccrrr}
\hline \multirow{2}{*}{ Methods } & coefficients $\hat{\beta}$ & & & & & \\
& $\hat{\beta}_{1}$ & $\hat{\beta}_{2}$ & $\hat{\beta}_{3}$ & $\hat{\beta}_{4}$ & $\hat{\beta}_{5}$ & $\hat{\beta}_{6}$ \\
\hline MAVE-B & 0.04695 & 0.58040 & 0.15398 & -0.43744 & 0.07417 & -0.45523 \\
MAVE-E & 0.08946 & 0.57626 & 0.23449 & -0.43922 & 0.05470 & -0.45003 \\
MAVE-G & 0.10508 & -0.64090 & -0.13815 & 0.55173 & -0.12048 & -0.33928 \\
\hline
\end{tabular}

Table 8: The Values of MSE and MAE for Single Index Model which are Estimated by Methods (MAVE-Biwieght, MAVE- Epanechnikov and MAVEGaussian) Based on Real Data

\begin{tabular}{lll}
\hline Methods & MSE & MAE \\
\hline MAVE-B & 0.00229 & 0.03518 \\
MAVE-E & 0.00233 & 0.03623 \\
MAVE-G & 0.00210 & 0.03171 \\
\hline
\end{tabular}

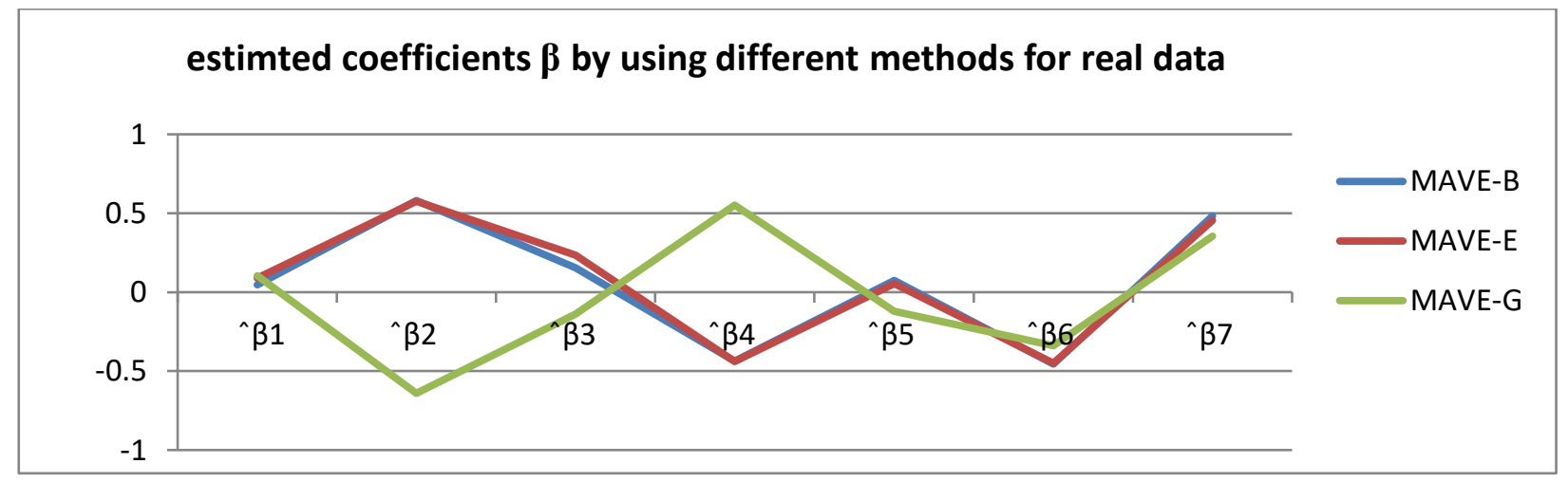

Fig. 1: Plot Explain the Estimated Coefficients B of Single - Index Model which are Estimated by the Different Methods (MAVE-B , MAVE-E and MAVE-G ) Based on A Real Data. 


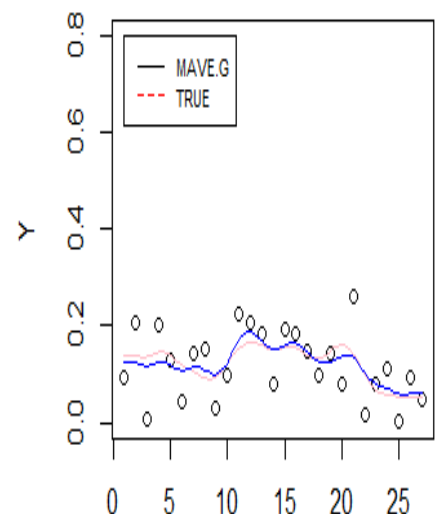

INDEX

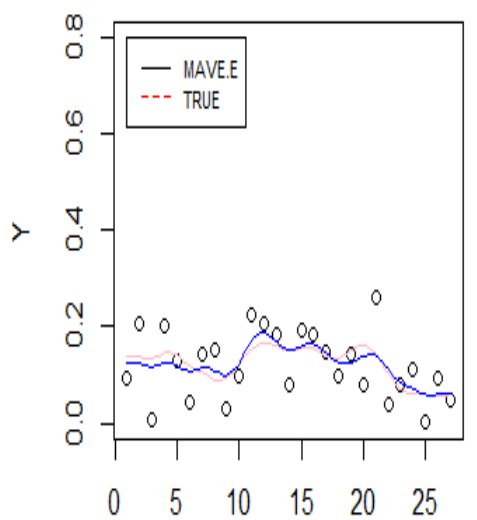

INDEX

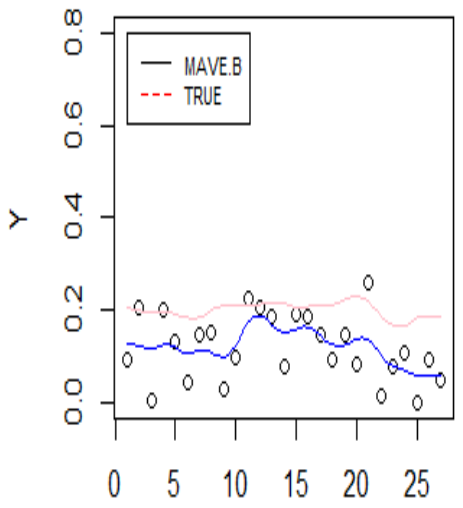

INDEX

Fig. 2: Plot for the Smooth Estimated Curve $\widehat{G}\left(X_{i}{ }^{t} \widehat{B}\right)$ of Single - Index Model which are Estimated By Methods (MAVE-B, MAVE-E and MAVE-G ) with Based on Areal Data.

According to the MSE and AME for link function $\hat{g}\left(\mathrm{X}_{\mathrm{i}}^{\mathrm{T}} \hat{\beta}\right)$ of single - index model from table 8 and figure 2 . We find that the same results in simulation study are extended to practical study, the results of the real data example confirm the results of the simulation studies, i.e,the MAVE-G show a better performance than the MAVE-E and MAVE-B method .

\section{Conclusions}

We can be conclude that the MAVE method based on Gaussian kernel function perform well in comparison to the MAVE method based on Epanechnikov and Biweight kernel function for estimated parameters and link function of semi-parametric single-index model.

\section{References}

[1] Al - kenani, A., and Yu, K. (2013), "Penalized single Indexquantile regression ". International Journal of statistics and probability, vol.2, No.3, pp. 12-30.

[2] Akkus, O. (2011), "Xplore package for the popular parametric and semi-parametric single index models " .Journe of science, vol.24, No.4, pp. 753-762 .

[3] Hansen, W.E. (2009),"lecture Notes on Non-parametric ".university of Wisconsin,spring.

[4] Hardle, W., Hall, P., and Ichimura, H. (1993)," optimal smoothing in single index models ". The Annals of statistics, vol.21, pp. 157178. https://doi.org/10.1214/aos/1176349020.

[5] Kong, E., Xia, Yi. (2007), "variable selection for the single index model".Biometrika94,pp.217-229. https://doi.org/10.1093/biomet/asm008.

[6] Kopytou, E., and Santalova, D. (2007), "Application of the single index model for forcasting of the inland conveyances "Recent advances in stochastic modeling and data analysis. Singupre, world scientific poblishing copte Ltd,pp.268-276. https://doi.org/10.1142/9789812709691_0033.

[7] Lai, P., Wang, Q., and Lion, H. (2011), "Bias-corrected GEE estimation and smooth-threshold GEE variable selection for single index models with clustered data ". Journal of multivariate analysis, vol.105,No.1,pp.422-432. https://doi.org/10.1016/j.jmva.2011.08.009.

[8] Leng, C.L., Xia, Y., \& Xu, J. (2008), “An adaptive estimation method for semi-parametric models and dimension reduction ". Department of statistics and Applied probability National university of Singapore .Exploration of a nonlinear world, pp. 347-360.

[9] Li , R. , and Fan , J., (2004), "New Estimation and model selection procedures for semi-parametric modeling in longitudinal Data A nalysis ", journal of the American statistical Association, vol .99, No . 467, pp. 710-723.

[10] Naik, P.A., and Tsai, C.L. (2001), "single index model selections". Biometrika88,pp.821-832. https://doi.org/10.1093/biomet/88.3.821.

[11] Peng, H., and Huang, T. (2011), "penalized least squares for single index models ". Journal of statistical planning and inference 141, pp. 1362-1379. https://doi.org/10.1016/j.jspi.2010.10.003.
[12] Thomas, J.F. (2006), "Simulation study for single index model" .submitted to the Department of Mathematical sciences of Clemson University, in partial fulfillment for The requirements for The degree of Master of science in Mathematical sciences.

[13] Wang, G. (2015),"High / Ultra High Dimensional single - index models ". A Dissertation submitted to The Graduate Faculty of The University of Georgina in partial Fulfillment of The requirements for the Doctor PHD. Department of statistics, Athens, GEORGIA.

[14] Wang ,Q.,Zhang,T.,and Hardle,W.,(2014), " An Extended single index model with missing response at random ". SFB 649, Humbodt, university $\mathrm{Zu}$ Berlin spandaure. http:// sfb649.wiwi.huberlin .de.

[15] Wang, T., Xu .P.,and Zhu , L.(2013), , Penalized Minimum Average Variance Estimation ". Statistics sinica 23, pp.543-569. https://doi.org/10.5705/ss.2011.275.

[16] Xia, Y. (2006), "Asymptotic Distribution for Tow estimators of the Single - index model", National university of singopore, Econometric Theory,22,pp.1112-1137. https://doi.org/10.1017/S0266466606060531. 